Revue d'histoire de l'Amérique française

ThEVUE D.HISTOIRE DE L'AMÉRIQUE FRANÇAISE

\title{
Liste des écrits de Saint Jean de Brébeuf
}

\section{René Latourelle}

Volume 3, numéro 1, juin 1949

URI : https://id.erudit.org/iderudit/801545ar

DOI : https://doi.org/10.7202/801545ar

Aller au sommaire du numéro

Éditeur(s)

Institut d'histoire de l'Amérique française

ISSN

0035-2357 (imprimé)

1492-1383 (numérique)

Découvrir la revue

Citer ce document

Latourelle, R. (1949). Liste des écrits de Saint Jean de Brébeuf. Revue d'histoire de l'Amérique française, 3(1), 141-147. https://doi.org/10.7202/801545ar d'utilisation que vous pouvez consulter en ligne.

https://apropos.erudit.org/fr/usagers/politique-dutilisation/ 


\section{BIBLIOGRAPHIE}

\section{LISTE DES ECRITS DE SAINT JEAN DE BREBEUF ${ }^{1}$}

Les écrits de saint Jean de Brébeuf forment un ensemble assez bigarré. On y trouve, en effet, des ouvrages de nature aussi différente que peuvent l'être un catéchisme, un dictionnaire, une Relation, un journal spirituel, des lettres à un supérieur majeur. Ils supposent la connaissance de trois langues - le français, le latin, le huron - et ils couvrent une période de dix-huit ans, soit de 1630 à 1648.

Pareille diversité ou, si l'on veut, pareil manque d'unité s'explique aisément chez un homme que la pensée d'un public aux écoutes ne troubla sans doute jamais. Un caractère commun distingue ses productions: ce sont moins des œuvres que des écrits. Et toujours ils sont justifiés par un devoir à remplir, un bien à opérer. Si Brébeuf compose un dictionnaire et une grammaire de la langue huronne, c'est pour venir en aide à ses compagnons d'apostolat. S'il nous laisse un journal spirituel, c'est que les supérieurs l'ont contraint. S'il compose une Relation, c'est que sa charge le demande ou que des motifs apostoliques le dirigent, comme celui de réchauffer le zèle missionnaire de la France ou de remercier les bienfaiteurs de la mission. Enfin, s'il écrit au P. Général, c'est à titre de Procureur, de Consulteur ou d'Admoniteur. Aucune place n'est faite à la gratuité. Chez Brébeuf, l'apôtre absorbe l'écrivain. Cet homme de Dieu redoute que la beauté ne le détourne du devoir. Et le devoir, pour lui, ce sont les âmes à sauver que le Christ lui a confiées.

A notre connaissance, il existe trois relevés des écrits de Brébeuf. Un premier, très incomplet, se trouve dans l'ouvrage de Sommer-

1. L'auteur a bien voulu nous permettre la publication de cette partie d'une bibliographie destinée à une thèse sur les Écrits de Saint Jean de Brébeuf. 
vogel: Bibliothèque de la Compagnie de Jésus. ${ }^{2}$ Sommervogel attribue à Brébeuf une traduction huronne du catéchisme du P. Ledesma, deux Relations - celles de 1635 et de 1636 - cinq lettres aux PP. Généraux de la Compagnie de Jésus et une grammaire huronne.

Un deuxième relevé se trouve au collège Sainte-Marie, ${ }^{3}$ établi par les PP. Félix Martin et Arthur Melançon. Désireux de presser devant les Congrégations romaines la cause de béatification des martyrs canadiens, ces deux archivistes s'appliquèrent à constituer pour elles un dossier aussi complet que possible. Leur liste signale tous les écrits actuellement connus, sauf quatre, distingue les autographes des apographes, leś textes conservés des textes perdus, mais ne date avec précision que les lettres aux PP. Généraux.

Le seul relevé d'allure vraiment scientifique que nous connaissions est celui de M. Lucien Lusignan paru en juin 1944 dans le Bulletin des recherches historiques. ${ }^{4}$ Ce relevé a le double mérite d'être précis et de signaler les ouvrages importants qui renferment tel ou tel des écrits de Brébeuf. Il reste cependant incomplet puisqu'il ignore sept des écrits conservés et passe sous silence tous les écrits perdus dont l'existence nous est cependant révélée par des passages des Relations.

Dans un relevé d'écrits, l'ordre chronologique semble devoir s'imposer comme le plus simple et le plus normal. Cet ordre, cependant, nous semble ici moins heureux puisqu'il ne coïncide pas avec celui que nous adoptons dans la suite de cette étude. Nous lui préférons donc un ordre plus logique, basé sur la nature même des écrits. De la sorte, nous pouvons grouper les écrits de Brébeuf sous les chefs suivants:

A: Relations et extraits de Relations.

B: Correspondance, latine et française.

C: Notes spirituelles.

D: Ouvrages en langue huronne.

E: Ecrits perdus.

Ce classement offre en outre l'avantage de réunir des textes dont la valeur d'authenticité est à peu près égale. Pour satisfaire néan-

2. C. Sommervogel, s.j., Bibliothèque de la Compagnie de Jésus (11 vols et 2 suppléments, 1890-1932), 2: col. 107-108.

3. ACSM 1516.

4. Lucien Lusignan, "Essai sur les écrits de deux martyrs canadiens", Le Bulletin des recherches historiques, 50 (1944): 174-192. 
moins aux exigences de la chronologie, nous respecterons, à l'intérieur de chaque groupement, l'ordre d'apparition des écrits. De plus, nous ferons suivre ce premier relevé d'un second, rétablissant l'ordre chronologique, et d'un troisième, insérant les écrits de Brébeuf dans le tableau de sa vie.

Au cours de notre étude, nous nous reporterons toujours au premier classement adopté.

\section{A: RELATIONS ET EXTRAITS DE RELATIONS.}

1: Relation/ de ce qui s'est passél' Aux Hurons,/ en l'année 1635./ Enuoyée à Kebec au Pere Le Ieune,/ par le P. Brebeuf. Lettre datée du 27 mai 1635, de la maison de Saint-Joseph au village d'Ihonatiria, publiée à Paris, chez Sébastien Cramoisy, en 1636, avec la Relation de 1635 du P. Paul Le Jeune, 113-206. II y eut aussi une édition de cette Relation en Avignon, chez Jaques Bramereau, ${ }^{5}$ en 1636, 337-392. Dans l'édition de Thwaites, ${ }^{6} \mathrm{JR}$, 8: 68-154. Dans l'édition de Québec, ${ }^{7}$ I, 1635: 23-42. Il existe une édition originale de la Relation de 1635 à la Bibliothèque du Parlement, à Ottawa, à la Bibliothèque Municipale de Montréal et à la Bibliothèque de l'Université Laval.

2: Relation / de ce qui s'est passédans le / Pays des Hurons /en l'année 1636. / Enuoyée à Kebec au R.P. Paul le Ieune / Supérieur de la Mission de la Compa-/gnie de Iesus, en la Nouuelle France. Lettre datée du 16 juillet 1636, de la Résidence de Saint-Joseph, au village d'Thonatiria. Elle forme la seconde partie de la Relation de 1636, publiée en 1637 chez Sébastien Cramoisy, 1-223. JR, 10: 6-316. Q 1 (1636): 76-139. Édition originale de la Relation de 1636 à la Bibliothèque du Parlement d'Ottawa, à la Bibliothèque Municipale de Montréal, à l'Université Laval de Québec, aux archives du Collège Sainte-Marie.

3: Mémoire sur le sauvage Sasousmat, surnommé Marsolet. Début de 1634. Mémoire envoyé par le P. Jean de Brébeuf au P. Paul Le Jeune et introduit par celui-ci dans la Relation de 1634 publiée en 1635 chez Sébastien Cramoisy, à Paris. Ce mémoire rapporte un baptême survenu en janvier 1634. Edition originale: 8-11; JR, 6: 108-112; Q 1 (1634): 3-4.

4: Lettre au P. Le Jeune. Ecrite du Long-Sault, ${ }^{8}$ en juillet 1634 par

5. James C. McCoy, Jesuit Relations of Canada, 1632-1673, a Bibliography (Paris, 1937), 32.

6. R.G. Thwaites, The Jesuit Relations and Allied Documents (73 vol., Cleveland, 1896-1901). Désormais, l'abréviation sera: JR.

7. Augustin Côté, ed., Relations des Jésuites (3 vol., Québec, 1858) A l'avenir, l'abréviation sera: $Q$.

8. Brébeuf, montant chez les Hurons en 1634, partit de Québec, le 1 er juillet (JR, 7: 212), fut aux Trois-Rivières, le 3 juillet (B: 2), et arriva au pays des Hurons, le 5 sout (JR, 8: 88). La lettre du Long-Sault est donc nécessairement du mois de juillet. 
le P. de Brébeuf et portée au P. Le Jeune par des Hurons. Insérée partiellement par celui-ci dans sa Relation de 1634, 331-333; JR, 7: 218-220; Q 1 (1634): 88-90.

5: Instruction pour les/ Peres de nostre Compagnie qui seront envoiéz aux Hurons. Conseils pratiques envoyés par le P. de Brébeuf au P. Le Jeune et cités par celui-ci dans sa Relation du 31 août 1637, imprimé à Rouen, en 1638, chez Jean Le Boulenger, 232-237; JR, 12: 116-122; Q 1 (1637): $71-72$.

6: Lettre au P. Paul Le Jeune, datée d'Ossossane, le 28 octobre 1637. Sorte de testament rédigé par Brébeuf, signé par lui et quatre de ses compagnons: les PP. Le Mercier, Chastellain, Garnier et Ragueneau. Destinée au P. Paul Le Jeune, supérieur des missions de la Compagnie de Jésus en Nouvelle-France. Cette lettre est citée par le P. François Joseph Le Mercier et fait partie de la Relation de 1638 imprimée à Paris, en 1638, chez Sébastien Cramoisy, 28-30; JR, 15: 60-64; Q 1 (1638): 43.

7: Voeu de ne jamais manquer à la grâce du martyre. Voeu en latin et en français cité par le P. Paul Ragueneau au chapitre cinquième de la Relation du 1er mai 1649 envoyêe au P. Jérôme Lalemant et imprimê à Paris, en 1650, chez Sébastien et Gabriel Cramoisy, 63-66. Date probable de ce voeu: $1639^{\circ}$. JR, 34: 164-168; Q II (1649): 18-19. Le chapitre cinquième de la Relation de 1649 fait aussi partie du Mémoire de $1652 .{ }^{10}$ Le voeu se trouve à la page 189-191. Il est enfin reproduit dans le Rapport de l'Archiviste de la Province de Québec pour l'année 1924-1925, 61-62.

8: Lettre au P. Jérôme Lalemant. Dans la Relation datée du 27 mai 1640, imprimée en 1641 à Paris, chez Sébastien Cramoisy, le P. Lalemant cite une partie d'une lettre du P. de Brébeuf dans laquelle celui-ci raconte les merveilles de la grâce chez une vieille femme et chez un nouveau baptisé, 93-97; JR,19: 200-206; Q I (1640): 76-77.

\section{B: Correspondance Latine et Francaise}

1: Lettre du P. Jean de Brébeuf, datée de Rouen, le 31 mai 1631, au P. Julien Perrault, S.J., à Caen. Texte français. Autographe de 27 lignes, adresse au verso, appartenant à la collection Philéas Gagnon. document 3821. Photographie de l'original aux archives du Collège Sainte-Marie, série des photostats. Lettre inédite.

2: Lettre du P. Jean de Brébeuf à un jésuite de Rouen. Datée des Trois-Rivières, le 3 juillet 1634. Texte français. Autographe de 15 lignes conservé aux archives du scolasticat des PP. Jésuites de Jersey, en Angleterre. Original photographié par le P. Léon Pouliot et conservé aux archives du collège Sainte-Marie, ACSM, E-26. Lettre inédite.

9. Félix Martin, s.j., Hurons et Iroquois, le P. Jean de Brébeuf, sa vie, ses travaux, son martyre (Paris, 1898), 259.

10. "Memoires touchant la / Mort et les Vertus / des Peres Isaac Jogues / Anne de Noue, Anthoine/ Daniel, Jean de Brebeuf/ Gabriel Lallement, charles garnier/ Noel Chabanel \& Un Seculier René Goupil, ACSM, 202. 
3: Lettre du P.Jean de Brébeuf au T. R.P. Mutius Vitelleschi, Général de la Compagnie de Jésus, à Rome. Texte latin. Ecrite de la Résidence Saint-Joseph, chez les Hurons, au bourg d'Ihonatiria, sans date. L'analyse de la lettre prouve à l'évidence, cependant, qu'elle est de 1636."11 Autographe conservé aux archives de la Compagnie de Jésus, à Rome. Gal. 109, I, ff.92, 92v, 93. Photographie de l'original au collège Sainto-Marie. Aussi copie de la lettre par le P. Félix Martin - ACSM, MS 102 - reproduite par Thwaites, JR, 11: 6-11. Traduction française dans Première Mission des Jésuites au Canada, lettres et documents inédits publiess par le P. Auguste Carayon. ${ }^{12}$

4: Lettre du P. Jean de Brébeuf au T. R.P. Mutius Vitelleschi, Gonéral de la Compagnie de Jésus, à Rome. Lettre Latine datée de la Résidence de Saint-Joseph, chez les Hurons, au bourg d'Ihonatiria, le 20 mai 1637. Autographe conservé aux archives de la Compagnie de Jésus, à Rome, Gal. 109, I, ff.67, 67v., 68, 68v. Photographie de l'original au collège SainteMarie. Dans Thwaites, JR,11: 12-20. Version française dans Carayon: $157-162$.

5: Lettre du P. Jean de Brébeuf au T. R.P. Mutius Vitelleschi, Général de la Compagnie de Jésus, à Rome. Lettre latine datée de la Résidence de Saint-Joseph, chez les Hurons, au bourg de Téanaostaiaé, le $13 \mathrm{mai}$ 1639. A utographe conservé aux archives de la Compagnie de Jésus, à Rome, Gal. 109, II, ff.353-353v, 354v. Photographie de l'original au collège Sainte-Marie. Lettre inédite. Version anglaise par Théodore Besterman. ${ }^{13}$

6: Lettre du P. Jean de Brébeuf au T. R.P. Mutius Vitelleschi, Général de la Compagnie de Jésus, à Rome. Lettre latine datée de Québec, NouvelloFrance, le 20 août 1641. Autographe conservé aux archives de la Compagnie de Jésus, à Rome, Gal. 109, II, ff. 365, 365v, 366, 366v, 367, 368v. Photographie de l'original au Collège Sainte-Marie. Texte latin inédit. Manuscrit en mauvais état, difficile à déchiffrer. Traduction anglaise dans Besterman, op. cit., 183-185. Quelques paragraphes dans Thwaites, JR,20: 102-104. Copie Martin, ACSM, MS 143.

7: Lettre du P. Jean de Brébeuf au T.R.P. Mutius Vitelleschi, Général de la Compagnie de Jésus, à Rome. Lettre latine datée de Québec, en Nouvelle-France, le ler octobre 1642. Autographe conservé aux archives de la Compagnie de Jésus, à Rome, Gal. 109, II, ff. 375, 375v, 376, 376v. Photo-

11. Plusieurs passages le font entendre nettement. Ainsi, Brébeuf écrit: "Depuis deux ans que nous sommes revenus ici, nous avons baptisé près de cent personnes". Or, Brébeuf est arrivé chez les Hurons en 1634 (JR, 8: 88). Il dit encore: "Nous n'étions ici l'année dernière que trois prêtres de la Compagnie; nous avons été cinq cette année". Or, la Relation de 1636 dit que le 13 aout 1635 sont arrivés les PP. Mercier et Pijart (JR, 10: 56). Ce qui fait bien cinq avec les PP. Brébeuf, Daniel, Davost qui s'y trouvent déjà.

12. Auguste Carayon, éd., Première Mission des Jésuites au Canada, Lettres et documents inédits, (Paris, 1864), 163-166. A l'avenir: Carayon.

13. Théodore Besterman, éd. et trad., The Travels and Sufferings of Father Jean de Brebeuf amony the Hurons of Canada, as described by himself, (London, 1938), 178-179. A l'avenir: Besterman. 
graphie de l'original au collège Sainte-Marie. Lettre inédite. Version anglaise dans Besterman: 186-187.

8: Lettre du P. Jean de Brébeuf au T. R.P. Mutius Vitelleschi, G6nóral de la Compagnie de Jøsus, à Rome. Lettre latine datée des TroisRivières, de la Résidence de la Conception, en la Nouvelle-France, le 23 septembre 1643. Autographe conservé aux archives de la Compagnie de Jessus, à Rome, Gal. 109, II, ff. 381, 381v, 382, 382v. Photographie de l'original aux archives du Collège Sainte-Marie. Dans Thwaites, JR, 23: 246-252. Version française dans Carayon: 222-225. Copie Martin, ACSM, MS 25.

9: Lettre du P. Jean de Brébeuf au T. R.P. Vincent Caraffa, Général de la Compagnie de Jésus, à Rome. Lettre latine datée de Sainte-Marie des Hurons, Nouvelle-France, le 2 juin 1648. Autographe conservé aux archives de la Compagnie de Jésus, à Rome, Gal. 109, II, ff. 402, 402v, 403, 403v. Manuscrit en mauvais état. Photographie de l'original au collège SainteMarie. Copie Martin, ACSM, MS 188. Dans Thwaites, JR,32: 59-64. Version française dans Carayon: 229-232.

\section{C: Notes Spirituelles}

1: Quelques Graces/Visions. Illustrations et autres/ Remarques extraictes des/ Manuscrits du P. Jean de Brébeuf. Ces notes intimes forment dix-sept pages d'un manuscrit, datant de 1652, conservé au Collège Sainte-Marie et connu sous le titre de Manuscrit de 1652. Le titre complet de ce manuscrit est: Mémoires touchant la/ Mort et les Vertus/des Peres Isaac Jogues/Anne de Noue, Anthoine/ Daniel, Jean de Brébeuf/Gabriel Lallement, charles garnier/ Noel Chabanel et Un Seculier/ René Goupil. De la page 224 à la page 240 de ce manuscrit sont groupées un certain nombre des notes intimes de Brébeuf. Ces notes sont datées. On trouve ces pages dans le Rapport de l'Archiviste de la Province de Québec pour l'année 1924-1925, 68-76. Dans l'édition miméographié du P. Arthur Melançon, 147-153.

2: Extraits des notes intimes du P. de Brébeuf. Au chapitre 5ème de la Relation de 1649, le P. Paul Ragueneau cite quelques textes qu'il déclare provenir des papiers du P. de Brébeuf. Ces textes, datés de 1634 et de 1639, n'apparaissent pas dans le groupe précédent. JR,34: 158—194.

\section{D: Ouvrages en Langue Huronne}

1: Doctrine chrétienne du R.P. Ledesme de la Compagnie de Jésus. Traduicte en langage Canadois autre que celuy des Montagnars, pour la conversion des habitans dudit pays. Par le R.P. Breboeuf de la mesme Compagnie. Catéchisme en quatorze leçons, paru d'abord à Rouen, chez Richard L'Allemant, en 1630, in-12, 26 pages. Puis inséré dans les vingt dernières pages de l'édition de 1632 de l'ouvrage de Samuel de Champlain intitulé: Les Voyages de la Nouvelle France occidentale, dicte Canada... \& un Catéchisme ou Instruction traduicte du françois au langage des peuples sauvages 
de quelque contrée... A Paris, chez Louis Seuestre, 1632. Texte français et huron en regard. En 1870, l'abbe C.H. Laverdière publia chez Desbarats, ̀̀ Québec, les Oeuvres de Champlain en 4 vols. Texte dans le 4ème vol. ou tome 6: $1393-1407$.

2: Iesous Ahatonhia, Noël huron, communément attribué à Brébeuf, sans date certaine. Texte huron, version anglaise et française, présentation et commentaires dans: The first Canadian Christmas Carol, Iesous Ahatonhia, Huron Indian Carol, circa 1641, by Father Jean de Brebeuf, english interpretation by J.E. Middleton, Toronto, 1927.

3: Prière en huron. Composée par Brébeuf et insérée par celui-ci dans sa Relation de 1636. Dans Thwaites, JR, 10: 68-72.

\section{E: Ecrits Perdus}

1: Lettre du P. Jean de Brébeuf au Récollet Joseph de la Roche d'Aillon, alors au pays des Neutres. Date probable: décembre 1626 et plutôt début de 1627. Sagard, dans son Histoire, ${ }^{14}$ cite une lettre du P. d'Aillon à un ami d'Angers, dans laquelle le Récollet parle d'une lettre de Brébeuf l'invitant à revenir du pays des Neutres, où il courait des dangers, au pays des Hurons.

2: Lettre du P. de Brébeuf au P. Paul le Jeune relative à un sauvage nommé Matchonon rencontré aux Trois-Rivières. Ecrite par Brébeuf en 1634, à son arrivée au pays des Hurons. Allusion à cette lettre dans la Relation de 1635 du P. Paul le Jeune. JR, 7: 276-277; Q I (1635): 6.

3: Dictionnaire et grammaire huronne. ${ }^{16}$ Allusions très nettes dans les Relations de 1636 et 1637. JR, 10: 54, 116; 14: 10. Aussi, JR, 6: 20.

4: Instructions et catéchismes en huron. Même époque. JR, 8: 142144; JR, 10: 32 .

Si nous faisons la somme des écrits de Brébeuf, nous arrivons donc à un total minimum de 25 écrits. Sur ce nombre, 21 sont encore conservés et 9 sont des autographes.

René latourelle, s.j.

14. Gabriel Sagard, Histoire du Canada et Voyages (Paris, 1636), livre IV, ch. 3,888 .

15. Ainsi, dans sa Relation de 1636, Brébeuf, parlant de l'été de 1635, dit: "Nous fismes un mémorial confus des mots que nous avions remarquez depuis nostre arrivée, \& puis nous esbauchasmes un Dictionnaire de la langue des Hurons, qui sera très profitable... Finalement nous nous occupasmes à reformer, ou plutost à ranger une grammaire". JR, 10: 54. Brébeuf consacre encore tout le chapitre quatrième de la 1ère partie de la Relation de 1636 à des observations sur la langue: "Ce n'est que pour en donner quelque petit avant-goust, \& en marquer quelques particularitez, attendant une grammaire et un Dictionnaire". JR, 10: 116. Daniel et Davost, les compagnons de Brébeuf étant en Huronie depuis un an seulement, on comprend que celui-ci soit l'auteur principal du dictionnaire et de la grammaire. 